\title{
Use of immunotherapy in the treatment of gastric cancer (Review)
}

\author{
LUHONG YANG $^{1,2}$, YANXIA WANG ${ }^{2}$ and HUAFENG WANG ${ }^{1,2}$ \\ ${ }^{1}$ Modern College of Humanities and Science, and ${ }^{2}$ School of Life Science, \\ Shanxi Normal University, Linfen, Shanxi 041004, P.R. China
}

Received May 2, 2018; Accepted April 29, 2019

DOI: $10.3892 / \mathrm{ol} .2019 .10935$

\begin{abstract}
Gastric cancer (GC) is a malignant tumor that negatively impacts human health, which typically presents in the advanced stages of disease in the majority of patients. Despite the development of combination chemotherapy, only a modest survival advantage is gained in patients with GC treated by this method. Recently, cancer immunotherapies have received considerable attention as a viable therapeutic option for GC. Specifically, the immune checkpoint inhibitors, chimeric antigen rector (CAR)-T cells and tumor vaccines, represent immunotherapies that have exhibited promising effects in the treatment of GC. A number of clinical trials have employed either immuno-oncology monotherapies or combination therapies to improve the overall survival time (OS) and objective response rate (ORR) of patients with GC. The current review presents a summary of the clinical effects of checkpoint inhibitors, including CAR-T and tumor vaccines, in the treatment of GC.
\end{abstract}

\section{Contents}

1. Introduction

2. Immune checkpoint inhibitors

3. CAR-T cell therapy

4. Tumor antigen vaccines

5. Conclusions

\section{Introduction}

Gastric cancer (GC) is a malignant tumor type, with wide-reaching impacts on human health. In 2016, there were $\sim 834,000 \mathrm{GC}$-associated mortalities worldwide (1). $\mathrm{GC}$ is the sixth most common type of cancer worldwide in

Correspondence to: Dr Huafeng Wang, School of Life Science, Shanxi Normal University, 1 Gongyuan Street,Linfen, Shanxi 041004, P.R. China

E-mail: wanghf@sxnu.edu.cn

Key words: gastric cancer, immunotherapy, immune checkpoint inhibitors, chimeric antigen receptor-T cell, tumor vaccine terms of incidence, with the second highest mortality rate among all types of cancer worldwide (1). In China, it has been estimated that there were 67,910 new cases of GC and 49,800 GC-associated mortalities in 2015 (2); therefore, GC is one of the most common types of cancer ranking second following lung cancer in terms of morbidity and mortality, and the 5-year survival rate of GC is only $28 \%$ (2). As the majority of patients with GC are primarily diagnosed at the advanced stages of disease, the potential for successful treatment is limited to a few patients where early detection and comprehensive treatment were achieved $(3,4)$. Even with use of combination chemotherapy, consisting of platinum and 5-fluorouracil (5-FU), only a modest survival advantage is obtained for patients with advanced GC (5-8). Therefore, the identification of novel therapeutics for the treatment of advanced GC represents an important area of investigation.

Immunotherapy is the treatment of disease by inducing, enhancing or suppressing an immune response (9). Immunomodulatory regimens often have fewer adverse side effects compared with the presently used drugs and include less potential for creating resistance when treating microbial diseases (10). Two types of cancer immunotherapies exist: Active and passive (11). Active immunotherapies utilize components of the patient's own immune system, in order to boost an immune response against tumors (11). Examples of active immunotherapies include chimeric antigen receptor (CAR)-T cell therapy and cancer vaccines (11). Passive immunotherapies utilize components of the immune system manufactured outside of the body, including monoclonal antibodies (11). Over the past 10 years, cancer immunotherapy has demonstrated to be surprisingly effective in the treatment of melanoma and has subsequently been applied to breast, prostate, kidney and lung cancer (12). Therefore, immunotherapy was designated as the breakthrough treatment of the year in 2013 (12).

The potential use of immunotherapy in GC has received a considerable amount of interest. The molecular features of GC have been analyzed by the Cancer Genome Atlas Research Network and four classes of tumor subtypes have been identified, including Epstein-Barr (EB) virus-positive tumors, microsatellite instability tumors, genomically stable tumors and tumors with chromosomal instability (13). In the EB virus subgroup, which accounts for $15 \%$ of GC tumors, the expression of programmed death-ligand-1 (PD-L1) is increased (13), which is indicative of the presence of stable immune cells and supports the use of an immune checkpoint inhibitor for the treatment of this GC subtype (13). 
In the present review, three principal types of cancer immunotherapies for GC were evaluated, including immune checkpoint inhibitors, adoptive cell therapy, including CAR-T cells, and tumor vaccines.

\section{Immune checkpoint inhibitors}

Introduction to immune checkpoint inhibitors. In tumor immunity, the immune system recognizes and eliminates tumor cells in order to inhibit tumor development (14). However, tumors can escape recognition and destruction by the immune system, thereby achieving a malignant reproduction regime (15). As the primary participant in the tumor immune response, the regulation of $\mathrm{T}$ lymphocyte activity can exert coordinated effects upon stimulatory and inhibitory molecules, with these inhibitory molecules serving as an immune checkpoint (16). The capacity for malignant tumors to escape immune surveillance involves the suppression of $\mathrm{T}$ cell activity and can occur through immune checkpoint pathways (Fig. 1A) $(17,18)$. As shown in Fig. 1A, the activity of $\mathrm{T}$ cells is inhibited by cytotoxic T lymphocyte-associated antigen-4 (CTLA-4) and programmed death 1 (PD-1)/PD-L1. Checkpoint inhibitor signaling pathways contribute to the anti-tumor effects of $\mathrm{T}$ cells, which achieve their goal of destroying tumor cells by inhibiting the capacity for tumor immune escape. As shown in Fig. 1B, checkpoint inhibitors combine with CTLA-4, PD-1 and PD-L1 to activate the immune response. Immune checkpoint inhibitors have previously been demonstrated to be effective in the treatment of a variety of malignant tumor types. As a result, utilization of immune intervention checkpoint signaling pathways has become a novel cancer treatment strategy $(19,20)$. In the past decade, three main types of checkpoint inhibitor immunotherapy drugs have been developed for use in preclinical and clinical studies. These drugs target CTLA-4, PD-1 or PD-L1 (21).

Anti-CTLA-4 antibodies. Ipilimumab is a monoclonal antibody that targets CTLA-4, which was approved by the USA Food and Drug Administration (FDA) for the treatment of advanced melanoma in 2011 (22). However, Ipilimumab has also been used in the treatment of numerous other types of cancer, including small-cell lung cancer (23). In a stage II clinical trial (NCT01585987) comparing Ipilimumab with that of standard care as administered immediately following first line chemotherapy in 114 patients with locally advanced (unresectable) or metastatic gastric and gastroesophageal junction cancer, no beneficial effects of Ipilimumab were observed (Table I) (24).

Tremelimumab is a monoclonal antibody that targets CTLA-4, which was approved as an orphan drug treatment for malignant mesothelioma on April 15, 2015 (25). The remission rate was $5 \%$ for 18 patients with gastric/gastroesophageal junction adenocarcinoma included in a phase II clinical trial (26); a rate that was below the second-line chemotherapy curative effect of cytotoxic drugs. Although the test of tremelimumab failed to achieve a pre-set remission rate in the final analysis, 4 patients achieved a stable condition and there was one case of partial relief, thereby demonstrating a continuing curative effect in remission, and such findings are promising with regard to the treatment of advanced GC.
Anti-PD-1 antibodies. Pembrolizumab, an IgG4 antibody, was the first monoclonal antibody to be developed that targets PD-1 (27). Pembrolizumab was approved by the USA FDA for the treatment of advanced non-small cell lung cancer in 2017 (28). The safety, tolerability and anti-tumor activity of pembrolizumab (also referred to as MK-3475) were assessed in patients with advanced triple negative breast cancer (TNBC), advanced head and neck cancer, advanced urothelial cancer and advanced GC in a phase I clinical trial (KEYNOTE-012/NCT01848834) (29). Of the 162 patients with advanced GC screened, $39 \mathrm{PD}-\mathrm{L1}^{+}$patients were enrolled in order to evaluate pembrolizumab. The results revealed that the overall response rate (ORR) was 33\% (95\% CI, 19-50) (29), the 6-month progression-free survival (PFS) and OS rates were 24 and $69 \%$, respectively. These results indicate that pembrolizumab exhibits a manageable degree of toxicity and promising antitumor activity in patients with advanced GC, with PD-L1 expression levels associated with the ORR.

Due to the encouraging results of the KEYNOTE-012 trial, numerous clinical trials involving inhibitory treatment of PD-1 for GC were initiated. The first study (30) was a phase II clinical trial of pembrolizumab as monotherapy and in combination with cisplatin +5 -FU in subjects with recurrent or metastatic gastric or gastroesophageal junction (G/GEJ) adenocarcinoma (KEYNOTE-059/NCT02335411) (31). Based on this research, pembrolizumab was approved as a third-line therapy for recurrent or metastatic G/GEJ cancer by the FDA in September 2017. In addition, there has been a phase III, randomized, open-label clinical trial (32) of pembrolizumab compared with paclitaxel in subjects with advanced gastric or gastroesophageal junction adenocarcinoma that progressed following first-line therapy with platinum and fluoropyrimidine (KEYNOTE-061) (33). Finally, a randomized, active-controlled, partially blinded, biomarker select, phase III clinical trial of pembrolizumab + cisplatin +5 -FU as a first-line treatment in subjects with advanced G/GEJ adenocarcinoma is ongoing (KEYNOTE-062) (34).

Currently in progress is a study involving a phase I dose escalation trial (NCT02268825) to assess the mean tolerated dose (MTD) of MK-3475 (pembrolizumab) in combination with mFOLFOX6, followed by a phase II expansion open label, nonrandomized trial with MK-3475 at the MTD in combination with mFOLFOX6 and supplemental celecoxib in patients with advanced gastrointestinal cancer. In this study, 128 cases of late-stage colorectal, stomach, esophagus, pancreas and biliary tract cancer are being assessed, which will be completed in 2020 .

In 2014, the FDA approved nivolumab, sold under the name Opdivo, which is a monoclonal antibody that targets PD-1 and is used for the treatment of advanced melanoma and lung squamous carcinoma (35). In 59 patients with advanced GC, nivolumab monotherapy was tested in the clinical trial Checkmate-032 (NCT02267343); the ORR was 17\%, and the 6-month PFS and OS rates were 18 and 49\%, respectively (36).

A study with 480 patients is in progress for a phase III trial (NCT02267343) to evaluate the efficacy and safety of ONO-4538 (nivolumab) in the standard treatment failure of unresectable advanced or recurrent GC, including esophagogastric junction cancer, which are refractory to or intolerant of standard therapy. Preliminary results revealed an improvement 
A

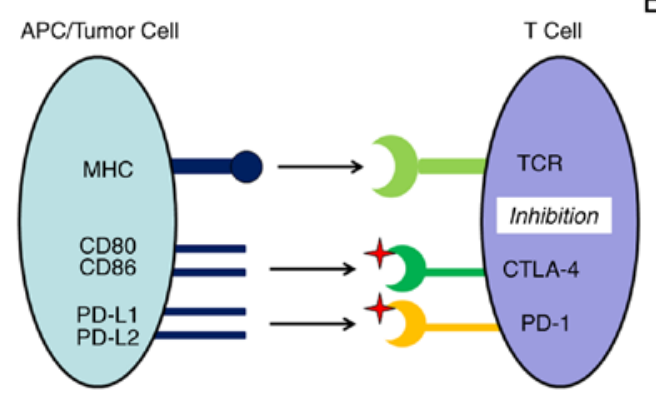

B

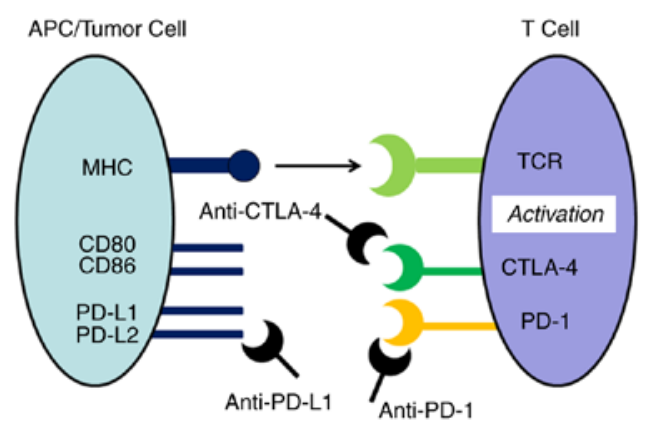

Figure 1. Principle of immune checkpoints and checkpoint inhibitors. (A) Tumor immune escape, which is the activity of T cells inhibited by the immune checkpoint signaling pathways, including CTLA-4 and PD-1/PD-L1. (B) Immune checkpoint inhibitors, which are monoclonal antibodies against immune system inhibitors, including CTLA-4 and PD-1, and its ligand PD-L1, activate an immune response. MHC, major histocompatibility complex; PD-L, programmed death-ligand; CTLA-4, cytotoxic T lymphocyte-associated antigen-4; PD-1, programmed death 1; TCR, T cell receptor; APC, antigen-presenting cell.

in survival with nivolumab treatment in advanced G/GEJ adenocarcinoma compared with placebo treatment (37).

In September 2017, based on a phase III study (ATTRACTION-02) of nivolumab in treating advanced gastric or gastroesophageal junction cancer in the Asian population in Japan, nivolumab was approved as a third-line treatment for unresectable or advanced G/GEJ (Table I) (38). This is the first time that a PD-1 monoclonal antibody has been approved for third-line treatment of advanced GC.

In addition, a phase I/II clinical trial (NCT01928394) is currently in progress to investigate the safety and efficacy of nivolumab as a single agent and in combination with ipilimumab in advanced solid tumors (39). The original project was designed to include six tumor types, including TNBC, GC, pancreatic adenocarcinoma, small cell lung cancer, bladder cancer and ovarian cancer; however, as of January 2017 only patients with bladder and pancreatic cancer have been recruited (Table I).

Anti-PD-L1 antibodies. To the best of our knowledge, three checkpoint inhibitor immunotherapy drugs against PD-L1 have been developed, including MDX-11-5, MEDI4736 and Avelumab. MDX-1105 (also known as BMS-936559) is a human monoclonal IgG4 antibody (40), and in a multicenter phase I trial (NCT00729664) (41), a total of 207 patients, including seven with GC, were treated with this anti-PD-L1 antibody. MDX-11-5 mediated the blockade of PD-L1, induced a durable tumor regression and prolonged stabilization of the disease; however, this was only observed in patients with non-small-cell lung cancer, melanoma or renal cell cancer. MDX-1105 was demonstrated to be relatively safe with regard to its use in the treatment of advanced GC (19).

At the 2015 American Society of Clinical Oncology meeting, Segal et al (42). reported their results from a phase I/II clinical trial (NCT01693562) with MEDI4736 (also referred to as durvalumab), a type of monoclonal antibody that is resistant to PD-L1. Their preliminary results, as obtained at only a 6-week median follow-up time period, indicated that MEDI4736 was effective against a variety of tumor types, including GC, where the PFS of two cases over a 24-week period was greater compared with that of the median PFS of stomach second-line therapy. The results from this small dataset (42) of patients demonstrated that concurrent palliative radiotherapy with the anti-PD-L1 durvalumab was well tolerated (43). To follow up on these findings and assess the efficacy and safety of MEDI4736 in combination with tremelimumab, a phase 1b/2 clinical study (NCT02340975) involving MEDI4736 and tremelimumab monotherapy in metastatic or recurrent gastric or gastroesophageal junction adenocarcinoma was initiated in January 2015 and is expected to be completed in 2019 (44).

As an engineered human monoclonal antibody targeting PD-L1, the safety and efficacy of avelumab were evaluated in a phase I, open-label, dose-escalation clinical trial (NCT01772004), which started in 2013 and was conducted in 1,758 cases with solid tumors, including 150 cases of stomach/stomach esophagus carcinoma (45). An additional phase I, open-label, dose-escalation clinical trial (NCT01943461) of avelumab is currently underway in Japanese patients with metastatic or locally advanced solid tumors, with plans to expand the study with the inclusion of Asian patients with GC, and the trial is expected to be completed by the end of June 2019 (46).

\section{CAR-T cell therapy}

Tumor cells exert an inhibitory effect upon the immune system, which restricts the induction and activation of the patient's autoimmune response (47). As an approach to mitigate this eventuality, immune lymphocytes are usually removed from the patient and trained to activate specific immune cells capable of recognizing cancer cells in vitro (48). Following amplification, these engineered cells can be re-infused into the patient to produce a rapid enhancement of the patient's immune function and destroy the tumor cells (49). Immune cells used in such training are primarily derived from lymphokine-activate killer cells, natural killer cells, cytotoxic T cells and tumor infiltrating lymphocytes (TILs), and this immunotherapy is referred to as adoptive cell therapy. Adoptive cell therapy is considered an active immunotherapy (11). CAR-T cell therapy is one of the most effective means for the treatment of malignant tumors $(49,50)$.

The core of CAR-T cells is the CAR, which includes three domains: i) The single chain fragment variable ( $\mathrm{scFv}$, binding tumor-associated antigen); iii) the extracellular hinge and transmembrane domain; and iii) the intracellular signaling domain (51). CARs can be introduced into $\mathrm{T}$ cells 


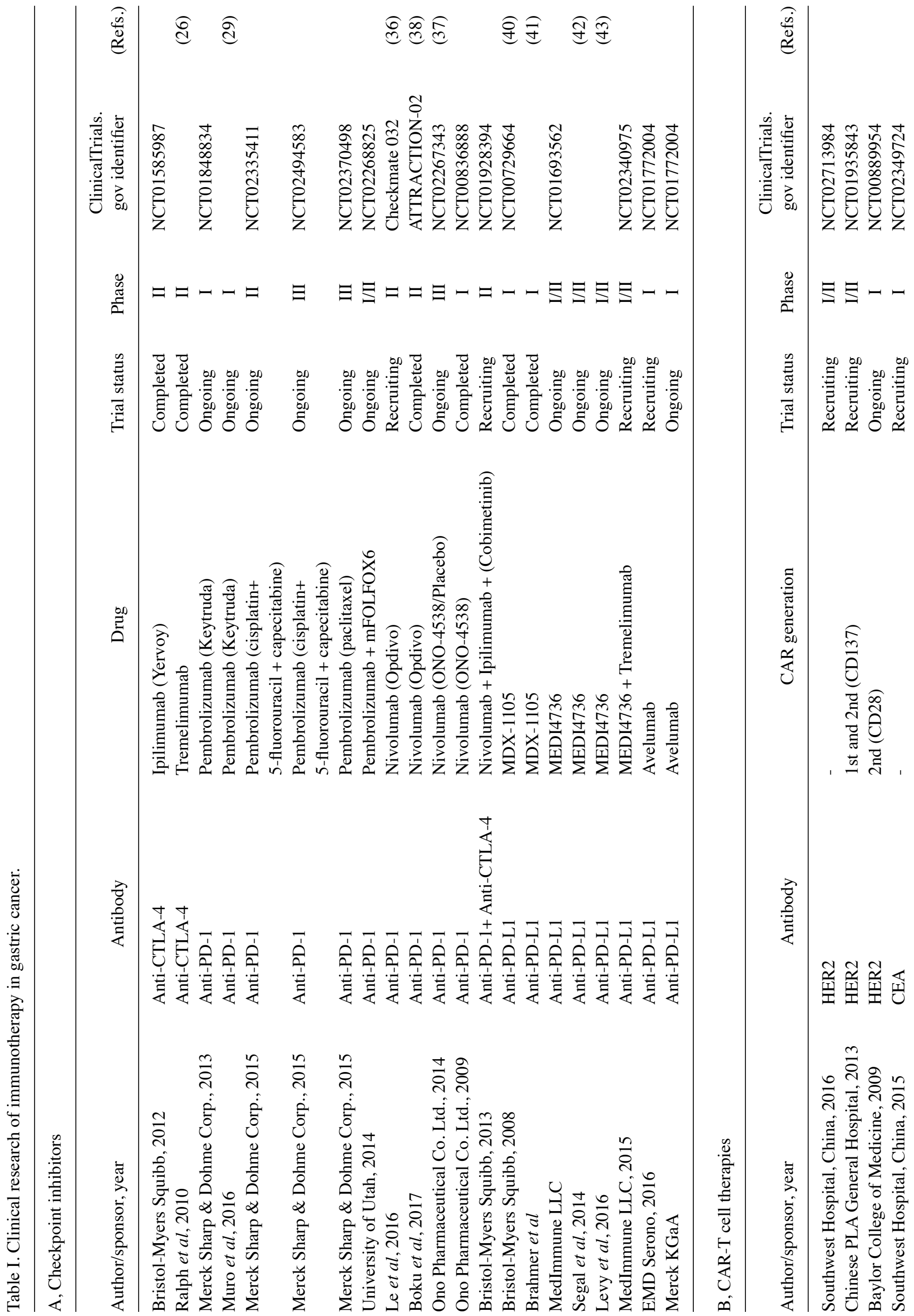




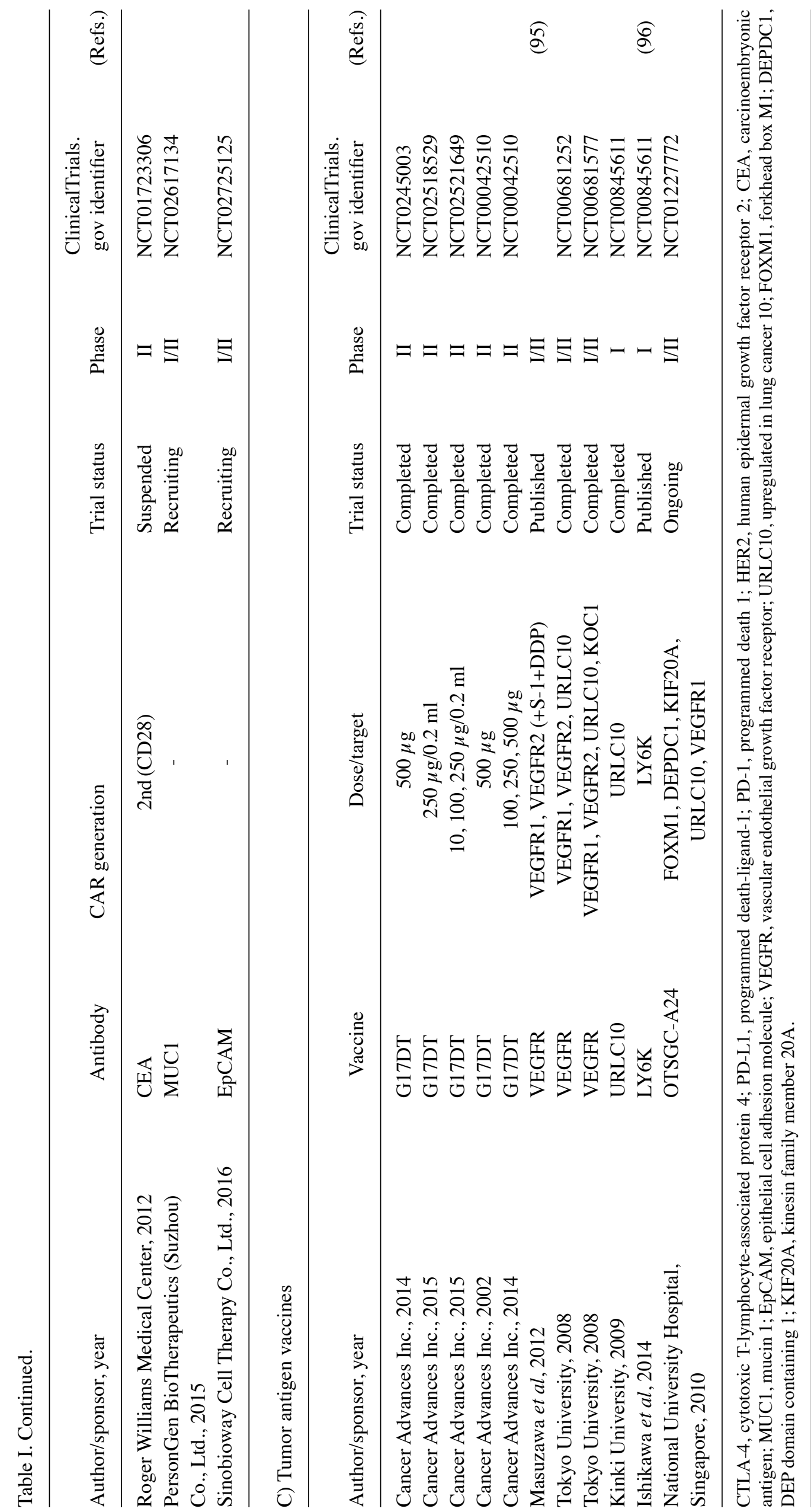


with high efficiency using viral vectors, and provide a means for recognition of cell surface components not restricted to major histocompatibility complexes (48). Therefore, $\mathrm{T}$ cells with CARs have the capacity to identify a broader range of cell surface components compared with the TCRs of natural $\mathrm{T}$ cells (Fig. 2). CAR-T technology represents a promising novel approach for the treatment of cancer. In July 2017, the CAR-T therapy CTL019, for use against relapsed/refractory acute lymphoblastic leukemia, was granted breakthrough therapy designation by the USA FDA (52). Currently, numerous biopharmaceutical companies are developing CAR-T technology for cancer therapy $(53,54)$.

CAR-T cell therapies have demonstrated efficacy against hematological malignancies, as demonstrated in a number of clinical trials (Table I). Recent studies have extended the application of CAR-T cell therapies for the treatment of solid tumors (55-59). Over the past decade, research has been directed at examining the efficacy of CAR-T therapy for use in gastrointestinal tumors, and antigens, including human epidermal growth factor receptor 2 (HER2), carcinoembryonic antigen (CEA), mucin 1 (MUC1) and epithelial cell adhesion molecule (EpCAM), have been used as targets in CAR-T therapy for GC (Table I).

HER2 is a proto-oncogene, which serves an important role in the pathogenesis and clinical development of gastric and gastroesophageal cancer (60-63). Investigators have developed anti-HER2 CAR-modified T cells and validated their efficiency in targeting HER2-positive cancers in preclinical studies (64). In order to establish the efficacy and adverse effects, and evaluate CAR-T cells persistence, tumor elimination and disease status following treatment, clinical studies (65-67) have been conducted with GC (Table I).

CEA is generally expressed in GC, and CEA-specific CAR-T cells can contribute to the delay of tumor growth and an extension in the survival of mice with GC (68). The primary purpose of the clinical trial NCT02349724 was to verify the safety of CEA-targeted CAR-T cells and to identify the proper dosage of CAR-T cells that should be infused (69).

MUC1 and EpCAM are transmembrane glycoproteins that are highly expressed in various types of cancer, including advanced prostate cancer, lung cancer and nasopharyngeal carcinoma (70-74). MUC1-specific CAR-T cells have previously been demonstrated to be effective in attacking MUC1-positive tumor cells (75); however, the altered expressions of EpCAM are associated with an aggressive biological response in GC (76). EpCAM-targeted CAR-T cells for stomach cancer will be evaluated in the phase I/II trials NCT02617134 and NCT02725125 starting in 2015 and estimated to be completed in November 2019, to assess the safety and efficacy of anti-MUC1 CAR-T cells for MUC1-positive relapsed or refractory solid tumors $(77,78)$.

\section{Tumor antigen vaccines}

A tumor antigen vaccine is a vaccine generated from cancer cells, portions of cancer cells or pure tumor antigens, which are isolated from tumor cells. A tumor antigen vaccine may stimulate the body's immune system to locate and destroy cancer cells (79). Sipuleucel-T (developed by Dendreon Corporation) was the first FDA-approved tumor vaccine,

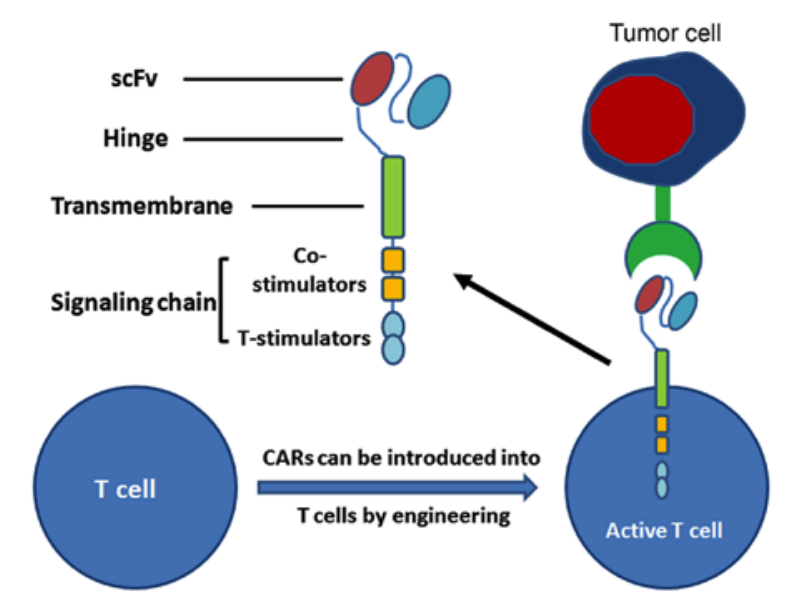

Figure 2. CARs and armoured CAR-T cells for antitumor therapy. T cells from a patient are modified by the introduction of predesigned CARs. When these $\mathrm{T}$ cells are re-infused into the patient, they can recognize and destroy malignant cells within the patient. CAR, chimeric antigen receptor.

approved in April 2010 for the treatment of asymptomatic or mild metastatic castration treatment of prostate cancer (80). Tumor vaccines mainly include whole-cell tumor vaccines, gene-modified tumor vaccines, dendritic cell vaccines, peptide vaccines and DNA vaccines (81). At present, thousands of tumor antigens that have been examined to evaluate their efficacy as antitumor agents and the antitumor activity of tumor peptide vaccines, such as G17DT, vascular endothelial growth factor receptor (VEGFR) and OTSGC-A24, have also been utilized in previous studies investigating GC (82-84).

G17DT is a vaccine that neutralizes gastrin-17, which is a hormone required for the growth of a number of cancer types of the gastrointestinal tract. G17DT has previously been demonstrated to be well tolerated and effective in the treatment of patients with advanced cancer $(85-87)$. Previous studies $(88,89)$ and a clinical trial (90) have been designed to investigate antibody responses to G17DT, dose-ranges, tolerability and safety in the treatment of patients with GC (Table I). In addition, results from a clinical phase II/III study (NCT00042510) revealed that following successful inoculation, G17DT induced specific and affinity antgastrin antibody (AGA), and AGA could inhibit proliferation and metastasis of tumors (89); therefore, G17DT combined with cisplatin and 5-FU could contribute to prolonging the median time-to-progression and median survival time for patients with unresectable adenocarcinoma of the stomach or gastroesophageal junction, compared with those without an anti-G17DT immune response (89). A report from the FDA, which designated G17DT as a fast track product on February 21, 2003, suggested that this agent may provide a way to improve the overall survival of stage IV GC when used in combination with cisplatin and 5-FU (91).

There are three main subtypes of VEGFR; 1,2 and 3 (92). Among them, VEGFR1 and VEGFR2 are two receptors for VEGF-A, and may serve as promising targets for anti-angiogenic immunotherapy against cancer in clinical settings $(93,94)$. In phase I/II studies (NCT00681252 and NCT00681577), the safety and time-to-progression of multiple-vaccine therapy of VEGFR1, VEGFR2 and upregulated in lung cancer 10 (URLC10) or VEGFR1, VEGFR2, URLC10 and KOC1 in treating patients with refractory GC 
have been evaluated. Masuzawa et al (95) investigated the ability for the peptide vaccine generated from VEGFR1 and VEGFR2 in combination with chemotherapy in one phase I/II study, and identified that a VEGF-specific cytotoxic lymphocyte reaction was induced. In this report, the efficiency reached $55 \%$ in 22 patients with advanced GC. In addition, $82 \%$ of the patients produced a cytotoxic $\mathrm{T}$ cell immune response to the two-peptide vaccines and exhibited an extension in the period of disease progression and increases in overall survival. In addition, in the NCT00845611 trial, the safety, and immunological and clinical responses of the URLC10 peptide vaccine were evaluated in patients with GC (Table I). On the basis of the same clinical trial registered with ClinicalTrial.gov, Ishikawa et al (96) demonstrated the immunogenicity of the LY6K-177 peptide vaccine in patients with advanced GC.

To improve the survival of patients with advanced GC, the safety and optimal dosing schedule of a cancer vaccine cocktail was evaluated in a phase I/II study (NCT01227772) with OTSGC-A24 targeting novel specific tumor antigens, including forkhead box M1, DEP domain containing 1, kinesin family member 20A, URLC10 and VEGFR1 (97).

\section{Conclusions}

Due to the notable success of immunotherapy in the treatment of different tumor types, including melanoma and lung squamous carcinoma $(98,99)$, the investigation of the therapeutic value of immunotherapy has expanded rapidly for application to other cancer types, including GS. However, the overall immunogenicity of gastric carcinoma is relatively weak and the immune treatment efficiency is quite limited in GC (13). Therefore, treatment with an immune checkpoint inhibitor is applicable for only a restrictive group of these patients, such as those with a gastric tumor subtype positive for the EB virus $(100,101)$.

After having established the effectiveness and safety of GC immunotherapy, the next critical issue to address involves selection of the best mode of treatment $(102,103)$. The investigation of targeted immune checkpoints has changed from that of single drug treatment to that involving a combination therapy, and this approach of applying immunotherapy in combination with chemotherapy has been adopted in numerous clinical settings (104). The majority of findings from studies on GC have demonstrated that, compared with chemotherapy alone, the combination of immunotherapy and chemotherapy improves the efficacy of treatment with varying degrees of success $(105,106)$. Nonetheless, due to the side effects associated with chemotherapy, the optimal protocol for combining chemotherapy with immunotherapy requires further investigation.

Immunotherapy was once considered to have little effect in common epithelial cancers with lower mutation rates $(107,108)$; however, a recent study demonstrated that adoptive transfer of autologous lymphocytes can produce widespread lytic activity against cancer cells (109). Zacharakis et al (110) reported a patient with breast cancer with adoptive transfer of four mutant-protein-specific TILs in conjunction with interleukin-2 and checkpoint blockade, which mediated the complete durable regression of metastatic breast cancer. The new immunotherapy approach is also expected to be used for the treatment of GC.

\section{Acknowledgements}

Not applicable.

\section{Funding}

This work was supported by the Key Discipline Construction of Shanxi Normal University (grant no. 0505/02100030) and the Shanxi Provincial University of Science and Technology Innovation Project (grant no. 20161107).

\section{Availability of data and materials}

All data generated or analyzed during this study are included in this published article.

\section{Authors' contributions}

LY collected and analysed the data, and was a major contributor in writing the original draft of the manuscript. YW collected and analysed data. HW reviewed and edited the manuscript.

\section{Ethics approval and consent to participate}

Not applicable.

\section{Patient consent for publication}

Not applicable.

\section{Competing interests}

The authors declare that they have no competing interests.

\section{References}

1. Global Burden of Disease Cancer Collaboration, Fitzmaurice C, Akinyemiju TF, Al Lami FH, Alam T, Alizadeh-Navaei R, Allen C, Alsharif U, Alvis-Guzman N, Amini E, et al: Global, regional, and national cancer incidence, mortality, years of life lost, years lived with disability, and disability-adjusted life-years for 29 cancer groups, 1990 to 2016: A systematic analysis for the global burden of disease study. JAMA Oncol 4: 1553-1568, 2018.

2. Chen W, Zheng R, Baade PD, Zhang S, Zeng H, Bray F, Jemal A, Yu XQ and He J: Cancer statistics in China, 2015. CA Cancer J Clin 66: 115-132, 2016.

3. TakeuchiC, Yamamichi N,ShimamotoT, Takahashi Y,MitsushimaT and Koike K: Gastric polyps diagnosed by double-contrast upper gastrointestinal barium X-ray radiography mostly arise from the Helicobacter pylori-negative stomach with low risk of gastric cancer in Japan. Gastric Cancer 20: 314-321, 2017.

4. Yu G, Torres J, Hu N, Medrano-Guzman R, Herrera-Goepfert R, Humphrys MS, Wang L, Wang C, Ding T, Ravel J, et al: Molecular characterization of the human stomach microbiota in gastric cancer patients. Front Cell Infect Microbiol 7: 302, 2017.

5. Fuchs CS, Tomasek J, Yong CJ, Dumitru F, Passalacqua R, Goswami C, Safran H, Dos Santos LV, Aprile G, Ferry DR, et al: Ramucirumab monotherapy for previously treated advanced gastric or gastro-oesophageal junction adenocarcinoma (REGARD): An international, randomised, multicentre, placebo-controlled, phase 3 trial. Lancet 383: 31-39, 2014.

6. Shen L, Xu JM, Feng FY, Jiao SC, Wang LW, Li J, Guan ZZ, Qin SK, Wang JJ, Yu SY, et al: Trastuzumab in combination with chemotherapy versus chemotherapy alone for first-line treatment of HER2-positive advanced gastric or gastroesophageal junction cancer: A Phase III, multi-center, randomized controlled trial, Chinese subreport. Zhonghua Zhong Liu Za Zhi 35: 295-300, 2013 (In Chinese). 
7. Galdy S, Cella CA, Spada F, Murgioni S, Frezza AM, Ravenda SP, Zampino MG and Fazio N: Systemic therapy beyond first-line in advanced gastric cancer: An overview of the main randomized clinical trials. Crit Rev Oncol Hematol 99: 1-12, 2016.

8. Bang YJ, Van CE, Feyereislova A, Chung HC, Shen L, Sawaki A, Lordick F, Ohtsu A, Omuro Y, Satoh T, et al: Trastuzumab in combination with chemotherapy versus chemotherapy alone for treatment of HER2-positive advanced gastric or gastro-oesophageal junction cancer (ToGA): A phase 3, open-label, randomised controlled trial. Lancet 376: 687-697, 2010.

9. Mellman I: Immunotherapies definition. Dictionary.com. Retrieved 2009-06-02. Nature 480: 480-489, 2011.

10. Masihi KN: Fighting infection using immunomodulatory agents. Expert Opin Biol Ther 1: 641-653, 2001.

11. Copp J, Xie WD, Zhang C and Berglin J: Immunotherapy and cell therapy for cancer. CJPT 30: 87-94, 2016.

12. Couzin-Frankel J: Breakthrough of the year 2013. Cancer immunotherapy. Science 342: 1432-1433, 2013.

13. Cancer Genome Atlas Research Network: Comprehensive molecular characterization of gastric adenocarcinoma. Nature 513 202-209, 2014.

14. Keir ME, Butte MJ, Freeman GJ and Sharpe AH: PD-1 and its ligands in tolerance and immunity. Annu Rev Immunol 26 : 677-704, 2008

15. Douglas $\mathrm{H}$ and Weinberg RA: Hallmarks of cancer: The next generation. Cell 144: 646-674, 2011.

16. Pardoll DM: The blockade of immune checkpoints in cancer immunotherapy. Nat Rev Cancer 12: 252-264, 2012.

17. Walker LS: Treg and CTLA-4: Two intertwining pathways to immune tolerance. J Autoimmun 45: 49-57, 2013.

18. Dolan DE and Gupta S: PD-1 pathway inhibitors: Changing the landscape of cancer immunotherapy. Cancer Control 21: 231-237, 2014.

19. Topalian SL, Hodi FS, Brahmer JR, Gettinger SN, Smith DC, McDermott DF, Powderly JD, Carvajal RD, Sosman JA, Atkins MB, et al: Safety, activity, and immune correlates of anti-PD-1 antibody in cancer. N Engl J Med 366: 2443-2454, 2012

20. Hodi FS, O'Day SJ, McDermott DF, Weber RW, Sosman JA, Haanen JB, Gonzalez R, Robert C, Schadendorf D Hassel JC, et al: Improved survival with ipilimumab in patients with metastatic melanoma. N Engl J Med 363: 711-723, 2010.

21. Jacob JA: Cancer immunotherapy researchers focus on refining checkpoint blockade therapies. JAMA 314: 2117-2119, 2015.

22. Ipilimumab. https://www.accessdata.fda.gov/scripts/ opdlisting/oopd/listResult.cfm.

23. Hellmann MD, Ott PA, Zugazagoitia J, Ready NE, Hann CL, De Braud FG, Antonia SJ, Ascierto PA, Moreno V, Atmaca A, et al: Nivolumab (nivo) \pm ipilimumab (ipi) in advanced small-cell lung cancer (SCLC): First report of a randomized expansion cohort from CheckMate 032. J Clin Oncol 35: 8503, 2017.

24. ClinicalTrials.gov: An efficacy study in gastric and gastroesophageal junction cancer comparing ipilimumab versus standard of care immediately following first line chemotherapy. https://clinicaltrials.gov/ct2/show/NCT01585987.

25. Tremelimumab. https://www.accessdata.fda.gov/scripts/ opdlisting/oopd/listResult.cfm.

26. Ralph C, Elkord E, Burt DJ, O'Dwyer JF, Austin EB, Stern PL, Hawkins RE and Thistlethwaite FC: Modulation of lymphocyte regulation for cancer therapy: A phase II trial of tremelimumab in advanced gastric and esophageal adenocarcinoma. Clin Cancer Res 16: 1662-1672, 2010.

27. Scapin G, Yang X, Prosise WW, McCoy M, Reichert P, Johnston JM, Kashi RS and Strickland C: Structure of full-length human anti-PD1 therapeutic IgG4 antibody pembrolizumab. Nat Struct Mol Biol 22: 953-958, 2015.

28. Garon EB, Rizvi NA, Hui R, Leighl N, Balmanoukian AS, Eder JP, Patnaik A, Aggarwal C, Gubens M, Horn L, et al Pembrolizumab for the treatment of non-small-cell lung cancer. N Engl J Med 372: 2018-2028, 2015.

29. Muro K, Chung HC, Shankaran V, Geva R, Catenacci D, Gupta S Eder JP, Golan T, Le DT, Burtness B, et al: Pembrolizumab for patients with PD-L1-positive advanced gastric cancer (KEYNOTE-012): A multicentre, open-label, phase 1b trial. Lancet Oncol 17: 717-726, 2016.

30. Bang YJ, Kang YK, Catenacci DV, Muro K, Fuchs CS, Geva R, Hara H, Golan T, Garrido M, Jalal SI, et al: Pembrolizumab alone or in combination with chemotherapy as first-line therapy for patients with advanced gastric or gastroesophageal junction adenocarcinoma: Results from the phase II nonrandomized KEYNOTE-059 study. Gastric Cancer 22: 828-837, 2019.
31. ClinicalTrials.gov: A study of pembrolizumab (MK-3475) in participants with recurrent or metastatic gastric or gastroesophageal junction adenocarcinoma (MK-3475-059/KEYNOTE-059). https://clinicaltrials.gov/ct2/show/NCT02335411.

32. Shitara K, Özgüroğlu M, Bang YJ, Di Bartolomeo M, Mandalà M, Ryu MH, Fornaro L, Olesiński T, Caglevic C, Chung HC, et al: Pembrolizumab versus paclitaxel for previously treated, advanced gastric or gastro-oesophageal junction cancer (KEYNOTE-061) A randomised, open-label, controlled, phase 3 trial. Lancet 392: 123-133, 2018.

33. ClinicalTrials.gov: A study of pembrolizumab (MK-3475) versus paclitaxel for participants with advanced gastric/gastroesophageal junction adenocarcinoma that progressed after therapy with platinum and fluoropyrimidine (MK-3475-061/KEYNOTE-061) https://clinicaltrials.gov/ct2/show/NCT02370498.

34. ClinicalTrials.gov: Study of pembrolizumab (MK-3475) as first-line monotherapy and combination therapy for treatment of advanced gastric or gastroesophageal junction adenocarcinoma (MK-3475-062/KEYNOTE-062). https://clinicaltrials. gov/ct2/show/NCT02494583

35. Nivolumab. https://www.accessdata.fda.gov/scripts/opdlisting/ oopd/listResult.cfm.

36. Le TD, Bendell JC, Calvo E, Kim JW, Ascierto PA, Sharma P, Ott PA, Bono P, Jeffry D, Evans TRJ, et al: Safety and activity of nivolumab monotherapy in advanced and metastatic $(\mathrm{A} / \mathrm{M})$ gastric or gastroesophageal junction cancer (GC/GEC): Results from the CheckMate-032 study. J Clin Oncol 34, 2016.

37. Kang YK, Boku N, Satoh T, Ryu MH, Chao Y, Kato K, Chung HC, Chen JS, Muro K, Kang WK, et al: Nivolumab in patients with advanced gastric or gastro-oesophageal junction cancer refractory to, or intolerant of, at least two previous chemotherapy regimens (ONO-4538-12, ATTRACTION-2): A randomised, double-blind, placebo-controlled, phase 3 trial. Lancet 390: 2461-2471, 2017

38. Boku N, Kang YK, Satoh T, Chao Y, Kato K, Chung HC, Chen JS, Muro K, Kang WK, Yoshikawa T, et al: 617OA Phase 3 Study of nivolumab (Nivo) in previously treated advanced gastric or gastroesophageal junction (G/GEJ) cancer: Updated results and subset analysis by PD-L1 expression (ATTRACTION-02). Ann Oncol 28, 2017.

39. ClinicalTrials.gov: A study of nivolumab by itself or nivolumab combined with ipilimumab in patients with advanced or metastatic solid tumors. https://clinicaltrials gov/ct2/show/NCT01928394.

40. Lee JY, Lee HT, Shin W, Chae J, Choi J, Kim SH, Lim H, Won Heo T, Park KY, Lee YJ, et al: Structural basis of checkpoint blockade by monoclonal antibodies in cancer immunotherapy. Nat Commun 7: 13354, 2016.

41. Brahmer JR, Tykodi SS, Chow LQ, Hwu WJ, Topalian SL, Hwu P, Drake CG, Camacho LH, Kauh J, Odunsi K, et al: Safety and activity of anti-PD-L1 antibody in patients with advanced cancer. N Engl J Med 366: 2455-2465, 2012.

42. Segal NH, Antonia SJ, Brahmer JR, Maio M, Blake-Haskins A, Vasselli XL, Ibrahim RA, Lutzky J and Khleif S: Preliminary data from a multi-arm expansion study of MEDI4736, an anti-PD-L1 antibody. J Clin Oncol 32: 3002, 2014

43. Levy A, Massard C, Soria JC and Deutsch E: Concurrent irradiation with the anti-programmed cell death ligand-1 immune checkpoint blocker durvalumab: Single centre subset analysis from a phase 1/2 trial. Eur J Cancer 68: 156-162, 2016.

44. A phase $1 \mathrm{~b} / 2$ study of MEDI4736 with tremelimumab, MEDI4736 or tremelimumab monotherapy in gastric or GEJ adenocarcinoma. https://clinicaltrials.gov/ct2/show/NCT02340975.

45. Chung HC, Arkenau HT, Lee J, Rha SY, Oh DY, Wyrwicz L, Kang YK, Lee KW, Infante JR, Lee SS, et al: Avelumab (anti-PD-L1) as first-line switch-maintenance or second-line therapy in patients with advanced gastric or gastroesophageal junction cancer: Phase $1 \mathrm{~b}$ results from the JAVELIN solid tumor trial. J Immunother Cancer 7: 30, 2019.

46. ClinicalTrials.gov: Avelumab in metastatic or locally advanced solid tumors (JAVELIN Solid Tumor JPN). https://clinicaltrials. gov/ct2/show/NCT01943461.

47. Mohme M, Riethdorf S and Pantel K: Circulating and disseminated tumour cells-mechanisms of immune surveillance and escape. Nat Rev Clin Oncol 14: 155-167, 2017.

48. Stauss HJ, Morris EC and Abken H: Cancer gene therapy with $\mathrm{T}$ cell receptors and chimeric antigen receptors. Curr Opin Pharmacol 24: 113-118, 2015.

49. Patel JM, Dale GA, Vartabedian VF, Dey P and Selvaraj P: Cancer CARtography: Charting out a new approach to cancer immunotherapy. Immunotherapy 6: 675-678, 2014. 
50. Gill S, Maus MV and Porter DL: Chimeric antigen receptor T cell therapy: 25 years in the making. Blood Rev 30: 157-167, 2016.

51. Zhang Q, Zhang Z, Peng M, Fu S, Xue Z and Zhang R: CAR-T cell therapy in gastrointestinal tumors and hepatic carcinoma: From bench to bedside. Oncoimmunology 5: e1251539, 2016.

52. Novartis CAR-T cell therapy CTL019 receives FDA breakthrough therapy designation for treatment of adult patients with $\mathrm{r} / \mathrm{r}$ DLBCL. https://www.novartis.com/news/media-releases/novartis-car-t-celltherapy-ctl019-receives-fda-breakthrough-therapy-designation.

53. Lee $\mathrm{YH}$ and Kim $\mathrm{CH}$ : Evolution of chimeric antigen receptor (CAR) T cell therapy: Current status and future perspectives. Arch Pharm Res, Mar 4, 2019 (Epub ahead of print).

54. Brudno JN and Kochenderfer JN: Recent advances in CAR T-cell toxicity: Mechanisms, manifestations and management. Blood Rev 34: 45-55, 2019.

55. Hege KM, Bergsland EK, Fisher GA, Nemunaitis JJ, Warren RS, McArthur JG, Lin AA, Schlom J, June CH and Sherwin SA: Safety, tumor trafficking and immunogenicity of chimeric antigen receptor (CAR)-T cells specific for TAG-72 in colorectal cancer. J Immunother Cancer 5: 22, 2017.

56. Lonez C, Verma B, Hendlisz A, Aftimos P, Awada A, Van Den Neste E, Catala G, Machiels JH, Piette F, Brayer JB, et al: Study protocol for THINK: A multinational open-label phase I study to assess the safety and clinical activity of multiple administrations of NKR-2 in patients with different metastatic tumour types. BMJ Open 7: e017075, 2017.

57. Tchou J, Zhao Y, Levine BL, Zhang PJ, Davis MM, Melenhorst JJ, Kulikovskaya I, Brennan AL, Liu X, Lacey SF, et al: Safety and efficacy of intratumoral injections of chimeric antigen receptor (CAR) $\mathrm{T}$ cells in metastatic breast cancer. Cancer Immunol Res 5: 1152-1161, 2017.

58. Zhang C, Wang Z, Yang Z, Wang M, Li S, Li Y, Zhang R, Xiong Z, Wei Z, Shen J, et al: Phase I escalating-dose trial of CAR-T therapy targeting CEA+ metastatic colorectal cancers. Mol Ther 25: 1248-1258, 2017.

59. Li J, Li W, Huang K, Zhang Y, Kupfer G and Zhao Q: Chimeric antigen receptor T cell (CAR-T) immunotherapy for solid tumors: Lessons learned and strategies for moving forward. J Hematol Oncol 11: 22, 2018.

60. Abrahao-Machado LF and Scapulatempo-Neto C: HER2 testing in gastric cancer: An update. World J Gastroenterol 22: 4619-4625, 2016.

61. Kurokawa Y, Matsuura N, Kimura Y, Adachi S, Fujita J, Imamura H, Kobayashi K, Yokoyama Y, Shaker MN, Takiguchi S, et al: Multicenter large-scale study of prognostic impact of HER 2 expression in patients with resectable gastric cancer. Gastric Cancer 18: 691-697, 2015.

62. Sheffield BS, Garratt J, Kalloger SE, Li-Chang HH, Torlakovic EE, Gilks CB and Schaeffer DF: HER2/neu testing in gastric cancer by immunohistochemistry: Assessment of interlaboratory variation. Arch Pathol Lab Med 138: 1495-1502, 2014.

63. Zulfiqar M, Bhalla A, Weindel M and Shidham VB: Molecular diagnostics in esophageal and gastric neoplasms. Clin Lab Med 33: 867-873, 2013.

64. Whilding LM and Maher J: ErbB-targeted CAR T-cell immunotherapy of cancer. Immunotherapy 7: 229-241, 2015.

65. A clinical research of CAR T cells targeting HER 2 positive cancer. https://clinicaltrials.gov/ct2/show/NCT02713984.

66. ClinicalTrials.gov: Treatment of chemotherapy refractory human epidermalgrowth factor receptor-2(HER-2) positive advanced solid tumors (CART-HER-2). https://clinicaltrials gov/ct2/show/NCT01935843.

67. ClinicalTrials gov: Her2 and TGFBeta cytotoxic T cells in treatment of Her2 positive malignancy (HERCREEM). https://clinicaltrials.gov/ct2/show/NCT00889954.

68. Wang L, Ma N, Okamoto S, Amaishi Y, Sato E, Seo N, Mineno J, Takesako K, Kato T and Shiku H: Efficient tumor regression by adoptively transferred CEA-specific CAR-T cells associated with symptoms of mild cytokine release syndrome. Oncoimmunology 5: e1211218, 2016.

69. Guest RD, Kirillova N, Mowbray S, Gornall H, Rothwell DG, Cheadle EJ,Austin E,Smith K, WattSM,Kühlcke K, et al: Definition and application of good manufacturing process-compliant production of CEA-specific chimeric antigen receptor expressing T-cells for phase I/II clinical trial. Cancer Immunol Immunother 63: 133-145, 2014.
70. Chalick M, Jacobi O, Pichinuk E, Garbar C, Bensussan A, Meeker A, Ziv R, Zehavi T, Smorodinsky NI, Hilkens J, et al: MUC1-ARF-A novel MUC1 protein that resides in the nucleus and is expressed by alternate reading frame translation of $\mathrm{MUC1}$ mRNA. PLoS One 11: e0165031, 2016.

71. Rajabi H, Hiraki M, Tagde A, Alam M, Bouillez A, Christensen CL, Samur M, Wong KK and Kufe D: MUC1-C activates EZH2 expression and function in human cancer cells. Sci Rep 7: 7481, 2017.

72. Genitsch V, Zlobec I, Thalmann GN and Fleischmann A: MUC is upregulated in advanced prostate cancer and is an independent prognostic factor. Prostate Cancer Prostatic Dis 19: 242-247, 2016.

73. Lee HK, Kwon MJ, Seo J, Kim JW, Hong M, Park HR, Min SK, Choe JY, Ra YJ, Jang SH, et al: Expression of mucins (MUC1, MUC2, MUC5AC and MUC6) in ALK-positive lung cancer: Comparison with EGFR-mutated lung cancer. Pathol Res Pract 215: 459-465, 2019.

74. Wang MH, Sun R, Zhou XM, Zhang MY, Lu JB, Yang Y, Zeng LS, Yang XZ, Shi L, Xiao RW, et al: Epithelial cell adhesion molecule overexpression regulates epithelial-mesenchymal transition, stemness and metastasis of nasopharyngeal carcinoma cells via the PTEN/AKT/mTOR pathway. Cell Death Dis 9: 2 , 2018.

75. Maher J and Wilkie S: CAR mechanics: Driving T cells into the MUC of cancer. Cancer Res 69: 4559-4562, 2009.

76. Warneke VS, Behrens HM, Haag J, Krüger S, Simon E, Mathiak M, Ebert MP and Röcken C: Members of the EpCAM signalling pathway are expressed in gastric cancer tissue and are correlated with patient prognosis. Br J Cancer 109: 2217-2227, 2013.

77. ClinicalTrials.gov: CAR-T cell immunotherapy in MUC1 positive solid tumor. https://clinicaltrials.gov/ct2/show/NCT02617134.

78. ClinicalTrials.gov: Study evaluating the efficacy and safety with CAR-T for stomach cancer (EECSC). https://clinicaltrials gov/ct2/show/NCT02725125.

79. Ott PA, Hu Z, Keskin DB, Shukla SA, Sun J, Bozym DJ, Zhang W, Luoma A, Giobbie-Hurder A, Peter L, et al: An immunogenic personal neoantigen vaccine for patients with melanoma. Nature 547: 217-221, 2017.

80. Cheever MA and Higano CS: PROVENGE (Sipuleucel-T) in prostate cancer: The first FDA-approved therapeutic cancer vaccine. Clin Cancer Res 17: 3520-3526, 2011.

81. Ribas A, Butterfield LH, Glaspy JA and Economou JS: Current developments in cancer vaccines and cellular immunotherapy. J Clin Oncol 21: 2415-2432, 2003.

82. Gilliam AD and Watson SA: G17DT: An antigastrin immunogen for the treatment of gastrointestinal malignancy. Expert Opin Biol Ther 7: 397-404, 2007.

83. Park DJ, Thomas NJ, Yoon C and Yoon SS: Vascular endothelial growth factor a inhibition in gastric cancer. Gastric Cancer 18: 33-42, 2015.

84. Sundar R, Rha SY, Yamaue H, Katsuda M, Kono K, Kim HS Kim C, Mimura K, Kua LF and Yong WP: A phase I/Ib study of OTSGC-A24 combined peptide vaccine in advanced gastric cancer. BMC Cancer 18: 332, 2018.

85. Watson SA, Michaeli D, Grimes S, Morris TM, Robinson G, Varro A, Justin TA and Hardcastle JD: Gastrimmune raises antibodies that neutralize amidated and glycine-extended gastrin-17 and inhibit the growth of colon cancer. Cancer Res 56: 880-885, 1996.

86. Smith AM, Justin T, Michaeli D and Watson SA: Phase I/II study of G17-DT, an anti-gastrin immunogen, in advanced colorectal cancer. Clin Cancer Res 6: 4719-4724, 2000.

87. Brett BT, Smith SC, Bouvier CV, Michaeli D, Hochhauser D, Davidson BR, Kurzawinski TR, Watkinson AF, Van Someren N, Pounder RE and Caplin ME: Phase II study of anti-gastrin-17 antibodies, raised to G17DT, in advanced pancreatic cancer. J Clin Oncol 20: 4225-4231, 2002.

88. Gilliam AD, Watson SA, Henwood M, McKenzie AJ, Humphreys JE, Elder J, Iftikhar SY, Welch N, Fielding J, Broome P and Michaeli D: A phase II study of G17DT in gastric carcinoma. Eur J Surg Oncol 30: 536-543, 2004.

89. Ajani JA, Hecht JR, Ho L, Baker J, Oortgiesen M, Eduljee A and Michaeli D: An open-label, multinational, multicenter study of G17DT vaccination combined with cisplatin and 5-fluorouracil in patients with untreated, advanced gastric or gastroesophageal cancer: The GC4 study. Cancer 106: 1908-1916, 2006. 
90. Rocha-Lima CM, de Queiroz Marques Junior E, Bayraktar S, Broome P, Weissman C, Nowacki M, Leslie M and Susnerwala S: A multicenter phase II study of G17DT immunogen plus irinotecan in pretreated metastatic colorectal cancer progressing on irinotecan. Cancer Chemother Pharmacol 74: 479-486, 2014.

91. Gastrin 17 vaccine-Aphton: Anti-gastrin 17 immunogen, G17DT. BioDrugs 17: 223-225, 2003.

92. Zhao T, Zhao W, Chen Y, Liu L, Ahokas RA and Sun Y: Differential expression of vascular endothelial growth factor isoforms and receptor subtypes in the infarcted heart. Int J Cardiol 167: 2638-2645, 2013.

93. Wada S, Tsunoda T, Baba T, Primus FJ, Kuwano H, Shibuya M and Tahara H: Rationale for antiangiogenic cancer therapy with vaccination using epitope peptides derived from human vascular endothelial growth factor receptor 2. Cancer Res 65: 4939-4946, 2005.

94. Ishizaki H, Tsunoda T, Wada S, Yamauchi M, Shibuya M and Tahara $\mathrm{H}$ : Inhibition of tumor growth with antiangiogenic cancer vaccine using epitope peptides derived from human vascular endothelial growth factor receptor 1 . Clin Cancer Res 12: 5841-5849, 2006.

95. Masuzawa T, Fujiwara Y, Okada K, Nakamura A, Takiguchi S, Nakajima K, Miyata $H$, Yamasaki M, Kurokawa $Y$ Osawa R, et al: Phase I/II study of S-1 plus cisplatin combined with peptide vaccines for human vascular endothelial growth factor receptor 1 and 2 in patients with advanced gastric cancer. Int J Oncol 41: 1297-1304, 2012.

96. Ishikawa $\mathrm{H}$, Imano $\mathrm{M}$, Shiraishi O, Yasuda A, Peng YF, Shinkai M, Yasuda T, Imamoto H and Shiozaki H: Phase I clinical trial of vaccination with LY6K-derived peptide in patients with advanced gastric cancer. Gastric Cancer 17: 173-180, 2014.

97. ClinicalTrials.gov: Study of OTSGC-A24 vaccine in advanced gastric cancer. https://clinicaltrials.gov/ct2/show/NCT01227772.

98. Valpione $\mathrm{S}$ and Campana LG: Immunotherapy for advanced melanoma: Future directions. Immunotherapy 8: 199-209, 2016

99. Stinchcombe TE: Unmet needs in squamous cell carcinoma of the lung: Potential role for immunotherapy. Med Oncol 31: 960, 2014.

100. Dong M, Wang HY, Zhao XX, Chen JN, Zhang YW, Huang Y, Xue L, Li HG, Du H, Wu XY and Shao CK: Expression and prognostic roles of PIK3CA, JAK2, PD-L1, and PD-L2 in Epstein-Barr virus-associated gastric carcinoma. Hum Pathol 53: 25-34, 2016

101. Shinozaki-Ushiku A, Kunita A and Fukayama M: Update on Epstein-Barr virus and gastric cancer (review). Int J Oncol 46 1421-1434, 2015.
102. Bolm L, Käsmann L, Paysen A, Karapetis C, Rades D, Wellner UF, Keck T, Watson DI, Hummel R and Hussey DJ: Multimodal anti-tumor approaches combined with immunotherapy to overcome tumor resistance in esophageal and gastric cancer. Anticancer Res 38: 3231-3242, 2018.

103. Lazăr DC, Avram MF, Romosan I, Cornianu M, Tăban S and Goldis A: Prognostic significance of tumor immune microenvironment and immunotherapy: Novel insights and future perspectives in gastric cancer. World J Gastroenterol 24: 3583-3616, 2018.

104. Cui J, Li L, Wang C, Jin H, Yao C, Wang Y, Li D, Tian H, Niu C, Wang G, et al: Combined cellular immunotherapy and chemotherapy improves clinical outcome in patients with gastric carcinoma. Cytotherapy 17: 979-988, 2015.

105. Wang Y, Wang C, Xiao H, Niu C, Wu H, Jin H, Yao C, He H, Tian $\mathrm{H}$, Han $\mathrm{F}$, et al: Adjuvant treatment combining cellular immunotherapy with chemotherapy improves the clinical outcome of patients with stage II/III gastric cancer. Cancer Med 6: 45-53, 2017.

106. Zhandossov O, Kaussova G and Koten A: Combined treatment for gastric cancer: Immunological approach. Turk J Gastroenterol 29: 151-156, 2018.

107. Hamanishi J, Mandai M, Ikeda T, Minami M, Kawaguchi A, Murayama T, Kanai M, Mori Y, Matsumoto S, Chikuma S, et al: Safety and antitumor activity of anti-PD-1 antibody, nivolumab, in patients with platinum-resistant ovarian cancer. J Clin Oncol 33: 4015-4022, 2015.

108. Le DT, Durham JN, Smith KN, Wang H, Bartlett BR, Aulakh LK, Lu S, Kemberling H, Wilt C, Luber BS, et al: Mismatch repair deficiency predicts response of solid tumors to PD-1 blockade. Science 357: 409-413, 2017.

109. Stevanović S, Pasetto A, Helman SR, Gartner JJ, Prickett TD, Howie B, Robins HS, Robbins PF, Klebanoff CA, Rosenberg SA and Hinrichs CS: Landscape of immunogenic tumor antigens in successful immunotherapy of virally induced epithelial cancer. Science 356: 200-205, 2017

110. Zacharakis N, Chinnasamy H, Black M, Xu H, Lu YC, Zheng Z, Pasetto A, Langhan M, Shelton T, Prickett T, et al: Immune recognition of somatic mutations leading to complete durable regression in metastatic breast cancer. Nat Med 24: 724-730, 2018.

This work is licensed under a Creative Commons Attribution-NonCommercial-NoDerivatives 4.0 International (CC BY-NC-ND 4.0) License. 